\title{
HIV-Therapie
}

\section{CHMP spricht sich für Zulassung eines Single-Tablet-Regimes aus}

_ Der Ausschuss für Humanarzneimittel (CHMP) der European Medicines Agency (EMA) hat sich für eine Zulassung des einmal täglichen Single-TabletRegimes aus Elvitegravir $150 \mathrm{mg}$, Cobicistat $150 \mathrm{mg}$, Emtricitabin $200 \mathrm{mg}$ und Tenofoviralafenamid (TAF) $10 \mathrm{mg}$ (Genvoya ${ }^{\circledR}$ zur Behandlung von HIV-1-Infektionen ausgesprochen - und zwar bei nicht vorbehandelten jugendlichen und erwachsenen HIV-Patienten und virologisch supprimierten erwachsenen $\mathrm{Pa}$ tienten, deren Therapie umgestellt wird, sowie bei Erwachsenen mit leichter bis mittelgradig eingeschränkter Nierenfunktion.

\section{Neuer Backbone}

TAF ist ein neuer Nukleotid-ReverseTranskriptase-Hemmer (NRTI), der eine hohe antivirale Effektivität bei einer zehnmal geringeren Dosis als Viread ${ }^{\circledast}$ (Tenofo- virdisoproxilfumarat, TDF) gezeigt hat. Darüber hinaus demonstrierte TAF in klinischen Studien in Kombination mit anderen antiretroviralen Wirkstoffen im Vergleich zu TDF eine Verbesserung bei Surrogat-Labor-Markern für Knochenund Nierenverträglichkeit.

Der Zulassungsantrag für E/C/F/TAF wird gestützt durch 48-Wochen-Daten aus zwei Zulassungsstudien der Phase III (Studie 104 und Studie 111), in denen für E/C/F/TAF bei nicht vorbehandelten erwachsenen HIV-Patienten eine NichtUnterlegenheit in Bezug auf die Virussuppression durch Stribild ${ }^{\bowtie}$ (Elvitegravir $150 \mathrm{mg} /$ Cobicistat $150 \mathrm{mg} /$ Emtricitabin $200 \mathrm{mg} / \mathrm{TDF} 300 \mathrm{mg}$ ) gezeigt werden konnte. In diesen Studien zeigte E/C/F/ TAF bessere Effekte auf die Knochenund Nierensicherheit im Vergleich zu Stribild ${ }^{\circledast}$. Der Zulassungsantrag wird zudem gestützt durch Daten aus zusätzli-

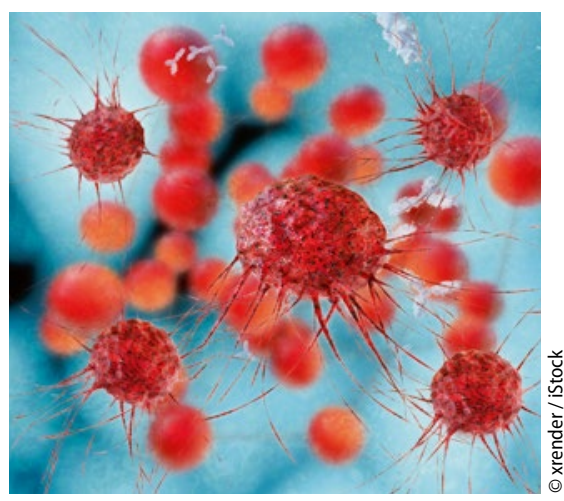

Humane Immundefizienz-Viren.

chen Phase-III-Studien bei Jugendlichen, bei virologisch supprimierten Erwachsenen, die aufE/C/F/TAF wechselten, sowie bei Erwachsenen mit leicht bis mittelgradig eingeschränkter Nierenfunktion.

- Red.

- Nach Informationen von Gilead

\section{Zullassung füir Edoxaban}

\section{Ein neuer Mitspieler im NOAK-Orchester}

_ Die neuen oralen Antikoagulanzien (NOAK) haben die Antikoagulation bei nicht-valvulärem Vorhofflimmern bzw. tiefen Venenthrombosen/Lungenembolien nicht nur einfacher, sondern auch sicherer gemacht. Mit dem Faktor-Xa-Inhibitor Edoxaban (Lixiana ${ }^{\circledR}$ ) steht jetzt ein neues NOAK zur Verfügung, das im Vergleich mit einem Vitamin-K-Antagonisten bei vergleichbarer Wirksamkeit mehr Sicherheit verspricht.

„Dies konnte die Substanz im Rahmen der ENGAGE-TIMI-48-Studie bei Patienten mit einem nicht-valvulärem Vorhofflimmern unter Beweis stellen“, sagte Prof. Andreas Goette, Paderborn. Die jährliche Rate an Schlaganfällen und pe- ripheren Embolien lag unter $1 \times 60 \mathrm{mg} / \mathrm{d}$ Edoxaban bei $1,18 \%$, unter Warfarin bei 1,5\%. Die Rate schwerer Blutungen war unter Edoxaban um 20\% niedriger.

Bei Patienten mit einer GFR von 30$50 \mathrm{ml} / \mathrm{min}$ oder einem Gewicht $\leq 60 \mathrm{~kg}$ oder bei einer Komedikation mit einem starken P-Glykoprotein-Inhibitor wurden nur $30 \mathrm{mg}$ verabreicht. „Das relative Risiko für schwere Blutungen war in dieser Patientengruppe größer als in der Gruppe ohne Dosisreduktion, und dies bei gleich guter Wirksamkeit", so Goette.

\section{Weniger Rezidive}

Ähnlich sind die Ergebnisse der HokusaiVTE-Studie bei Patienten mit tiefer Ve- nenthrombose bzw. Lungenembolie, wobei die Patienten flexibel für 3-12 Monate entweder mit Warfarin oder $60 \mathrm{mg}$ Edoxaban behandelt wurden. Bei der Verhinderung eines symptomatischen Rezidivs erwies sich Edoxaban als dem Warfarin nicht unterlegen, aber das Risiko relevanter Blutungen war signifikant um 19\% niedriger. „Bei Patienten mit Lungenembolie und rechtsventrikulärer Dysfunktion war die Rezidivrate unter Edoxaban aber um fasst 50\% niedriger", so PD Dr. Ingo Ahrens, Freiburg.

\section{Dr. Peter Stiefelhagen}

- Pressekonferenz "Zulassung für LIXIANA ${ }^{\infty}$ - Neues orales Antikoagulanz"; München, Juli 2015 (Veranstalter: Daiichi Sankyo) 